\section{RMD Open}

Rheumatic \& Musculoskeletal Diseases

\title{
Allergic conditions and risk of rheumatoid arthritis: a Swedish case- control study
}

\author{
Vanessa L Kronzer (10 , ${ }^{1}$ Helga Westerlind (1) , ${ }^{2}$ Lars Alfredsson, ${ }^{2}$ \\ Cynthia S Crowson (D) , ${ }^{3}$ Lars Klareskog (D) , ${ }^{4}$ Marie Holmqvist (D) , \\ Johan Askling (1D) ${ }^{2}$
}

To cite: Kronzer VL, Westerlind $\mathrm{H}$, Alfredsson $\mathrm{L}$, et al. Allergic conditions and risk of rheumatoid arthritis: a Swedish case-control study. RMD Open 2022;8:e002018. doi:10.1136/ rmdopen-2021-002018

- Additional supplemental material is published online only. To view, please visit the journal online (http://dx.doi.org/10. 1136/rmdopen-2021-002018).

Received 11 0ctober 2021 Accepted 25 January 2022

Check for updates

(C) Author(s) (or their employer(s)) 2022. Re-use permitted under CC BY-NC. No commercial re-use. See rights and permissions. Published by BMJ.

${ }^{1}$ Rheumatology, Mayo Clinic, Rochester, Minnesota, USA ${ }^{2}$ Clinical Epidemiology Unit, Dept of Medicine Solna, Karolinska Institutet, Stockholm, Sweden ${ }^{3}$ Department of Quantitative Health Sciences, Mayo Clinic, Rochester, Minnesota, USA ${ }^{4}$ Rheumatology, Karolinska Institutet, Stockholm, Sweden

Correspondence to Dr Vanessa L Kronzer; kronzer.vanessa@mayo.edu

\section{ABSTRACT}

Objective To determine the association of allergic conditions with incident rheumatoid arthritis (RA), especially in relation to smoking history and anticitrullinated peptide antibody (ACPA) status.

Methods This case-control study included 3515 incident RA cases and 5429 matched controls from the Epidemiological Investigation of Rheumatoid Arthritis study 1995 to 2016, including questionnaire-based information on eight allergic conditions composed from a list of 59 unique allergies. We used logistic regression and adjusted ORs (aOR) to assess the association between allergic conditions and risk of RA, adjusting for age, sex, residential area, body mass index, education, and smoking, and stratified by smoking and ACPA.

Results A history of any reported allergy was equally common in $\mathrm{RA}(\mathrm{n}=1047,30 \%)$ as among population controls ( $n=1540,29 \%$ ), aOR $1.04,95 \% \mathrm{Cl} 0.95$ to 1.15. Metal, respiratory, food, plant/pollen and chemical allergies were not associated with risk of RA. By contrast, statistically significant associations were observed for animal dander allergy ( $6 \%$ vs $5 \%$, aOR $1.37,95 \% \mathrm{Cl} 1.03$ to 1.82), especially in ACPA-positive RA (aOR $1.4695 \% \mathrm{Cl}$ 1.06 to 2.01) and for atopic dermatitis, in particular for older and ACPA-negative RA (aOR 2.33, 95\% $\mathrm{Cl} 1.37$ to 3.96 at age 80 ). Never smokers with allergic rhinitis also had increased risk of developing RA (aOR $1.30,95 \% \mathrm{Cl}$ 1.00 to 1.68).

Conclusion Most common allergies do not increase risk of RA, nor do they protect against RA. However, some allergic conditions, notably animal dander allergy, atopic dermatitis and allergic rhinitis, were associated with an increased risk for RA.

\section{INTRODUCTION}

Increasing evidence suggests that rheumatoid arthritis (RA) may originate in mucosal sites such as the lungs and oral cavity. Smoking is a strong risk factor for RA, and is believed to trigger RA through generation of anticitrullinated peptide antibodies (ACPA). ${ }^{12}$ Asthma has been shown to be associated with RA in several studies, ${ }^{3-8}$ at least in never smokers. ${ }^{910}$ Two recent retrospective studies

\section{Key messages}

What is already known about this subject?

- Some studies have suggested an association between atopic diseases and rheumatoid arthritis (RA).

What does this study add?

- Most of the allergic conditions studied, including food allergies, were not associated with increased risk of RA.

- However, we identified animal dander as a novel risk factor for RA-especially anticitrullinated peptide antibody (ACPA)-positive RA.

- Atopic dermatitis was associated with RA in ACPAnegative RA and/or older adults, and allergic rhinitis was associated with increased risk of RA in never smokers.

How might this impact on clinical practice or further developments?

- Based on these findings, clinicians can reassure patients that allergic conditions do not highly increase risk of $\mathrm{RA}$, though reducing animal dander exposure might be helpful for those at highest risk.

have also demonstrated an association between atopic diseases and incident RA, ${ }^{3} 6$ one using self-reported definition of allergies ${ }^{3}$ and the other using diagnosis codes. ${ }^{6}$ In the latter, the association between allergies and RA was stronger with history of more than one allergic disease. ${ }^{6}$ These findings call for assessments of atopic disease in relation to smoking and ACPA status. They also raise the question of whether other allergic conditions, such as allergic rhinitis, contact and food allergies, also increase RA risk.

Food allergies have been linked with RA by historical case reports of RA sustained remission after food allergy treatment, ${ }^{11}{ }^{12}$ a study of rats showing increased egg and milk $\operatorname{IgE}$ in rats with $\mathrm{RA}^{13}$ and a recent retrospective case-control study using self-reported food 
allergy. ${ }^{3}$ However, another study that measured food allergy by skin prick test showed no association with RA. ${ }^{14}$ Similarly, another study showed no association of grass allergy assessed by skin prick test with RA. ${ }^{14}{ }^{15}$ Additional but limited evidence from retrospective studies shows no association between self-reported allergy to grass, ${ }^{3}$ house mites, ${ }^{16}$ pets ${ }^{3}$ or insects ${ }^{3}$ with RA. Other allergy types such as metal and chemical allergies have not been studied.

To address these gaps, we leveraged Epidemiological Investigation of Rheumatoid Arthritis (EIRA), a population-based study of incident RA in Sweden ${ }^{17}$ to determine the relationship between allergic conditions and risk of RA, taking smoking and ACPA status into account.

\section{METHODS}

\section{Study design}

This case-control study involved 3515 incident RA cases and 5429 controls recruited to the EIRA study between 1995 and 2016, within 1 year since RA symptom onset. Controls were randomly selected from the national population and matched 1:1 until 2005 and thereafter 2:1 on age, sex and residential area. All participants completed a questionnaire at the time of enrolment, along with written informed consent. Response rate for the first phase of EIRA (until 2005) was 96\% for cases and $82 \%$ for controls as described previously. ${ }^{18}$ Response rate for the second phase of EIRA (since 2005) is $91 \%$ for cases and $69 \%$ for controls.

\section{Exposure (allergic conditions)}

Participants self-reported allergic conditions from a list of 59 potential allergic exposures on the EIRA enrolment questionnaire. The questionnaire specifically asked whether they had history of hay fever (allergic rhinitis) or eczema (atopic dermatitis). The remaining allergy types were reported from a list of options, but only on the questionnaires administered 2005 and prior. We combined these 59 options into six additional allergy categories. Animal dander allergy asked if the participant had any fur allergies (including cat). Metal allergies included nickel, cobalt, chrome, silver or gold. Respiratory allergies included any report of effort asthma, nonexertional asthma, cold asthma, non-allergic asthma or 'other allergic airway disease.'Food allergies included any report of allergy to seafood, kernel fruits/nuts, gluten, citrus, fruits/vegetables, eggs, chocolate, lactose, alcohol, milk, poultry, wine/liquor, fish, soy, rye, blue cheese or legumes. Plant/pollen allergies included pollen, flowers, fir, mould, eucalyptus or hyacinth allergies. Chemical allergies included cosmetics, perfume, ink, solvent, tobacco smoke, detergent, skin cream, shampoo, plastic, 'various chemicals', plaster, rubber, amalgam, ammonia, latex, charcoal, cement, conditioner, concrete colour, chlorine, dish soap, hair dye, or formaldehyde. To reflect an 'allergic phenotype' across conditions, we created an 'any allergy' (yes/no) variable combining presence of any above the allergic exposures. We also created a variable quantifying the number of reported allergic conditions (categorised as $0,1,2,3+$ ) out of the 59 potential allergic exposures.

\section{Outcome (RA)}

Inclusion criteria for EIRA included adults age 18 years or older with RA diagnosed for the first time. Exclusion criteria included inability to speak Swedish and age $>70$ years (until 2009). All RA cases were examined and diagnosed by a rheumatologist at the time of enrolment and fulfilled American College of Rheumatology/EULAR 1987 or 2010 criteria for RA. ${ }^{19}{ }^{20}$ ACPA status was determined using the commercial CCP2 diagnostic kit from Eurodiagnostica for frozen baseline sera from EIRA patients, while rheumatoid factor (RF) status was determined at inclusion by the recruiting site. RA cases could have positive ACPA, RF or both.

\section{Covariates}

Covariates including age, sex, residential area (ten geographic areas), body mass index (BMI), educational level (compulsory school only, upper secondary school or university degree) and smoking (never, non-regular, past and current) were self-reported by participants at the time of the EIRA enrolment questionnaire. 'Ever' smokers included non-regular, past and current smokers.

\section{Statistical analysis}

$\chi^{2}$ tests compared proportions, and Wilcoxon rank-sum tests with medians and IQR compared continuous variables. We performed separate, unconditional logistic regression models of each allergic condition to obtain adjusted ORs (aOR) for all RA, ACPA-positive RA and ACPA-negative RA, adjusting for age, sex, residential area, BMI, education, and smoking (never, non-regular, past and current). Appropriately powered logistic regression models require at least 70 events for this number of covariates, ${ }^{21}$ which was easily met by the 3515 RA cases in this study. For each allergic condition, we also tested interactions with age, sex, and smoking because of observed differences in RA phenotypes by these variables and included them in the model if they were statistically significant $(p<0.05)$. We then repeated all models for RF-positive RA. Finally, we evaluated the duration (in years) that any allergy overall occurred before RA as a predictor of RA. Only $111(1.24 \%)$ of the participants were missing data for one or more of the covariates (age, sex, residential area, BMI, education or smoking), and these participants were excluded from further analysis. All analyses were prespecified in a protocol and performed using SAS V.9.4 (SAS Institute).

\section{RESULTS}

Among the 3515 RA cases included in this study, median age was 55 (IQR 45-63) years, 71\% were female, 2262 $(66 \%)$ were positive for ACPA and $2301(66 \%)$ were positive for RF. Compared with their 5429 matched controls, 
Table 1 Characteristics of the EIRA RA cases from 1995 to 2016 and population controls at enrolment questionnaire

\begin{tabular}{|c|c|c|c|}
\hline Characteristic & RA cases $(\mathrm{N}=3515)$ & Controls ( $\mathrm{N}=5429)$ & Adjusted OR ${ }^{\star}$ for all RA $(95 \% \mathrm{Cl})$ \\
\hline Age, years, median (Q1,Q3) & $55(45,63)$ & $56(44,63)$ & 1.00 (0.99 to 1.00$)$ \\
\hline Female sex, N (\%) & $2493(71)$ & $3838(71)$ & 1.05 (0.95 to 1.16$)$ \\
\hline \multicolumn{4}{|l|}{$\mathrm{BMI}, \mathrm{kg} / \mathrm{m}^{2}, \mathrm{~N}(\%)$} \\
\hline$<20$ & $252(7)$ & $320(6)$ & $1.23(1.03$ to 1.48$)$ \\
\hline $20-25$ & $1574(45)$ & $2576(48)$ & (ref) \\
\hline $25-30$ & $1178(34)$ & $1811(34)$ & 1.04 (0.94 to 1.15$)$ \\
\hline $30+$ & $502(14)$ & $699(13)$ & 1.12 (0.98 to 1.28$)$ \\
\hline \multicolumn{4}{|l|}{ Educational level, N (\%) } \\
\hline Compulsory school only & $876(25)$ & $1043(19)$ & (ref) \\
\hline Upper secondary school & $1803(51)$ & $2649(49)$ & $0.80(0.71$ to 0.89$)$ \\
\hline University degree & $835(24)$ & $1730(32)$ & 0.61 (0.53 to 0.69$)$ \\
\hline \multicolumn{4}{|l|}{ Smoking status, N (\%) } \\
\hline Never smoker & $1146(33)$ & $2413(45)$ & (ref) \\
\hline Non-regular smoker & $287(8)$ & $478(9)$ & $1.26(1.07$ to 1.49$)$ \\
\hline Past smoker & $1146(33)$ & $1562(29)$ & $1.56(1.40$ to 1.73$)$ \\
\hline Current smoker & $915(27)$ & $924(17)$ & 1.95 (1.73 to 2.19$)$ \\
\hline
\end{tabular}

*Adjusting for age, sex, BMI, education, smoking. Bold values are statistically significant $(p<0.05)$.

BMI, body mass index; EIRA, Epidemiological Investigation of Rheumatoid Arthritis; RA, rheumatoid arthritis.

RA cases had lower BMI and educational level, and were more often smokers (table 1 ).

A total of 1047 (30\%) of RA cases and $1540(29 \%)$ of the controls reported a history of at least one of the allergies studied, with 226 (6\%) RA cases and $382(7 \%)$ controls reporting two allergic conditions and $140(4 \%)$ RA cases and 208 (4\%) controls reporting three or more. The most common allergic conditions were atopic dermatitis ( $11 \%$ of cases and $10 \%$ of controls), plant/pollen allergies (9\% of cases and $10 \%$ of controls), and allergic rhinitis ( $9 \%$ of cases and $8 \%$ of controls) (table 2 ).
After adjustment, a history of any allergic condition was not associated with risk of RA (aOR 1.04, 95\% CI 0.95 to 1.15 ). Having two (aOR $0.93,95 \%$ CI 0.78 to 1.11 ) or three or more (aOR 1.05, 95\% CI 0.84 to 1.32 ) allergic conditions was also not associated with increased risk of RA. Metal, respiratory, food, plant/pollen and chemical allergies were not associated with RA (table 2). Animal dander was the only allergy associated with risk of RA overall, and mostly for ACPA positive RA (table 2). In addition, atopic dermatitis was associated with increased risk of ACPA-negative RA (aOR 1.32, 95\% CI 1.06 to

Table 2 Association between types of allergies and risk of RA among the 3515 EIRA cases diagnosed with RA between 1995 and 2016 and and their 5429 controls

\begin{tabular}{|c|c|c|c|c|c|}
\hline \multirow[b]{2}{*}{ Allergy type } & \multicolumn{2}{|l|}{ No (\%) } & \multicolumn{3}{|c|}{ Adjusted* OR $(95 \% \mathrm{Cl})$} \\
\hline & $\begin{array}{l}\text { RA cases } \\
(\mathrm{N}=3515)\end{array}$ & $\begin{array}{l}\text { Controls } \\
(\mathrm{N}=5429)\end{array}$ & $\begin{array}{l}\text { All RA } \\
(\mathrm{N}=3515)\end{array}$ & $\begin{array}{l}\text { ACPA +RA } \\
\text { (N=2262) }\end{array}$ & $\begin{array}{l}\text { ACPA- RA } \\
\text { (N=1165) }\end{array}$ \\
\hline Animal dander† & $89(6)$ & $134(5)$ & 1.37 (1.03 to 1.82$)$ & 1.46 (1.06 to 2.01$)$ & 1.17 (0.73 to 1.88$)$ \\
\hline Allergic rhinitis & $270(9)$ & $387(8)$ & $1.30(1.00$ to 1.68$) \ddagger$ & 1.24 (0.90 to 1.70$) \ddagger$ & 1.39 (0.97 to 2.00$) \ddagger$ \\
\hline Atopic dermatitis & $345(11)$ & $453(10)$ & $1.16(0.98$ to 1.25$) \S^{*}$ & 0.99 (0.83 to 1.18$)$ & $1.32(1.06$ to 1.65$) \S^{*}$ \\
\hline Metal allergy $\dagger$ & $43(3)$ & $58(2)$ & 1.27 (0.84 to 1.91$)$ & 1.23 (0.77 to 1.98$)$ & 1.54 (0.83 to 2.83 ) \\
\hline Respiratory allergy $\dagger$ & $154(11)$ & $271(9)$ & 1.11 (0.90 to 1.38$)$ & 1.15 (0.90 to 1.47$)$ & 0.98 (0.69 to 1.39$)$ \\
\hline Food allergy† & $19(1.4)$ & $40(1.5)$ & 1.01 (0.58 to 1.76$)$ & 1.04 (0.55 to 1.97$)$ & 1.04 (0.43 to 2.48$)$ \\
\hline Plants and pollen $\dagger$ & $130(9)$ & $293(10)$ & 0.94 (0.75 to 1.18$)$ & $0.96(0.74$ to 1.24$)$ & 0.91 (0.64 to 1.31$)$ \\
\hline Allergy to chemicals $\dagger$ & $24(1.8)$ & $51(1.9)$ & 0.85 (0.51 to 1.40$)$ & 0.81 (0.45 to 1.48$)$ & 0.82 (0.36 to 1.83$)$ \\
\hline
\end{tabular}

${ }^{*}$ Adjusting for age, sex, residential area, body mass index, education and smoking. Bold values are statistically significant $(p<0.05)$.

†Data only available for the first version of questionnaire, 2006 and prior, $\mathrm{N}=1884 \mathrm{RA}$ cases and $\mathrm{N}=2146$ controls.

†Interaction term with smoking was significant $(p<0.05)$. OR shown assumes most common scenario (never smoker).

§Interaction term with age was significant $(p<0.05)$. OR shown assumes median age ( 55 years).

ACPA, anticitrullinated peptide antibodies; EIRA, Epidemiological Investigation of Rheumatoid Arthritis; RA, rheumatoid arthritis. 
Table 3 Association of allergic conditions with RF positive RA among the EIRA cases from 1995 to 2016

\begin{tabular}{lccc} 
& $\mathbf{N}(\%)$ or median (IQR) & & \\
\cline { 2 - 3 } Allergic condition & $\mathbf{R F}+\mathbf{R A}$ cases (N=2301) & Controls (N=5429) & Adjusted* OR (95\% Cl) for RF +RA \\
\hline Animal dander & $62(7)$ & $134(5)$ & $\mathbf{1 . 4 7}(\mathbf{1 . 0 6}$ to 2.03) \\
Allergic rhinitis & $180(9)$ & $387(8)$ & $1.35(0.99$ to 1.84$) \dagger$ \\
Atopic dermatitis & $227(11)$ & $453(10)$ & $1.04(0.87$ to 1.23$)$ \\
Metal allergy & $28(3)$ & $271(9)$ & $1.25(0.78$ to 2.00$)$ \\
Respiratory allergy & $106(11)$ & $40(1.5)$ & $1.18(0.92$ to 1.51$)$ \\
Food allergy & $12(1.3)$ & $293(10)$ & $1.00(0.52$ to 1.95$)$ \\
\hline Plants and pollen & $90(10)$ & $51(1.9)$ & $1.03(0.79$ to 1.33$)$ \\
\hline Allergy to chemicals & $15(2)$ & $0.83(0.46$ to 1.51$)$ \\
\hline
\end{tabular}

${ }^{*}$ Adjusting for age, sex, residential area, body mass index, education and smoking. Bold values are statistically significant $(p<0.05)$. †Interaction term with smoking was significant $(p<0.05)$. OR shown assumes most common scenario (never smoker). EIRA, Epidemiological Investigation of Rheumatoid Arthritis; RA, rheumatoid arthritis; RF, rheumatoid factor.

1.65). While atopic dermatitis was not associated with RA risk overall (aOR $1.16,95 \%$ CI 0.98 to 1.25 ), interaction analyses indicated a statistically significant association with RA for individuals aged 60 years or higher at RA diagnosis (online supplemental table S1). For example, at age 20 years, the aOR for all RA was 0.67 (95\% CI 0.46 to 0.98 ), whereas at age 80 years, the aOR for all RA was 1.71 (95\% CI 1.17 to 2.51) and for ACPA-negative RA was aOR 2.33 (95\% CI 1.37 to 3.96). Similarly, allergic rhinitis was not associated with RA in smokers (aOR $0.89,95 \% \mathrm{CI}$ 0.72 to 1.09 ), but was associated among never smokers (aOR 1.30 , 95\% CI 1.00 to 1.68 ).

The association of allergic diseases with risk of RF-positive RA was nearly identical to that of ACPA-positive RA (table 3). The duration in years that any allergy (combined, overall) occurred before RA did not impact its association with RA (aOR $0.98,95 \%$ CI 0.92 to 1.05 ). Characteristics of participants missing any of the covariates were similar to those with complete data except that those with missing data had a slightly lower educational level and were more likely to be never smokers (online supplemental table S2).

\section{DISCUSSION}

This population-based case-control study of incident RA demonstrated that overall, allergic diseases were not associated with risk of RA. However, we identified animal dander allergy as a novel risk factor for RA, especially ACPA-positive RA. In addition, atopic dermatitis was associated with RA, though this association was confined to ACPA-negative RA and/or older adults. Finally, allergic rhinitis was associated with increased risk of RA in never smokers.

The lack of association between metal, respiratory, plant/pollen and chemical allergies and RA risk is novel to this study. Metal and chemical allergies had never been studied before. Asthma has been associated with increased risk of $\mathrm{RA}^{3}{ }^{4}$ including in this population, ${ }^{10}$ but non-asthma respiratory allergies including cold and effort asthma had not yet been studied. Two prior studies showed no association between grass allergy and RA, ${ }^{3}{ }^{15}$ supporting this study's observed lack of association between plant/pollen allergies and RA. The lack of association between food allergy and RA was initially surprising given prior studies suggesting an association. ${ }^{3}{ }^{11-13}$ However, all of those were cross-sectional studies of prevalent rather than incident RA as done in this study. ${ }^{311-13}$ Furthermore, one only asked participants about shellfish and nut allergy, ${ }^{3}$ one was an animal study using $\operatorname{IgE}$ as a proxy for food allergy, ${ }^{13}$ and one was a case series of four patients, ${ }^{11}$ raising questions about their validity. In addition, another study also showed no association between food allergy and RA. ${ }^{14}$ Overall, the lack of association between many allergens and RA can provide reassurance to patients and clinicians.

Our observed association between animal dander allergy and incident RA, especially ACPA-positive RA, was a novel finding to this study. One prior study investigated pet allergy and RA and found no association. ${ }^{3}$ However, that study found a similar positive direction of association (OR 1.15 in the incident cohort), had only 175 incident RA cases, and did not divide RA patients by ACPA status. Furthermore, horse exposure has recently been associated with increased risk of granulomatosis with polyangiitis. ${ }^{22}$ The mechanism for such associations between animal exposure and rheumatic disease may involve respiratory irritation, as respiratory diseases have been associated with $\mathrm{RA}^{10}$ and other rheumatic diseases such as inflammatory myopathies. ${ }^{23}$ While this novel finding will require further replication, it represents a potentially modifiable risk factor for individuals at high risk of RA.

We noted a weak association between allergic rhinitis and RA. While many cross-sectional studies of allergic rhinitis and prevalent RA have been performed with mixed results, the two prospective studies of incident RA did show a positive association like this study. ${ }^{56}$ The particularly strong association among never smokers is unique to our study. One reason for the negative association 
between allergic rhinitis and smoking could be collider stratification bias, where conditioning on a common effect (ie, RA) induces a negative correlation between its risk factors. ${ }^{24}$ Nevertheless, among the two studies of allergic rhinitis reporting smoking, the one with the lower prevalence of smoking $(46 \%)$ reported a strong association with RA, ${ }^{25}$ while the one with a relatively high prevalence of smoking $(64 \%)$ reported no association. ${ }^{26}$ Our recent study also showed that respiratory diseases, including chronic upper respiratory diseases such as allergic rhinitis, were associated with increased risk of RA predominately in never smokers. ${ }^{10}$

We also found an association between atopic dermatitis and RA for older adults as well as ACPA-negative RA. Historically, several studies showed no association between atopy and RA, but all were small, studied prevalent RA and did not control for confounders. ${ }^{14} 16$ 27-30 More recent studies have shown a positive association between atopic diseases and RA. ${ }^{3625} 31$ The association with atopic dermatitis in our study is supported by two other recent studies showing pre-existing atopic dermatitis was associated with incident RA. ${ }^{52} \mathrm{~A}$ third study found a statistically non-significant association with atopic dermatitis, but did find older-aged women with multiple allergic conditions had increased risk of RA. ${ }^{6}$ The association with ACPA-negative RA could indicate misclassification of diseases such as psoriatic arthritis. Nevertheless, the association between atopic dermatitis, age and ACPAnegative RA merits further exploration.

The bidirectional association between certain atopic and autoimmune diseases supports a possible association between atopic diseases and RA that merits further study. For example, in one study, RA was associated with increased risk of developing allergic conditions such as asthma and allergic rhinitis. ${ }^{25}$ Similarly, autoimmune disease in parents increases risk of allergic disease in children. ${ }^{33}$ Shared environmental risk factors like smoking, overlapping genetic risk ${ }^{34}$ or shared immune dysregulation $^{35}$ such as cytokine activation ${ }^{36}$ may all play a role in this bidirectional association. Epithelial barrier disruption via could represent another shared mechanism. Indeed, skin disruption from atopic dermatitis and/or filaggrin mutations increase risk of other allergic diseases such as allergic rhinitis and asthma in a process known as 'atopic march,' and could similarly sensitise the immune system to autoimmune diseases. ${ }^{37}$ Investigating genetic variants and gene by environment interactions in these individuals with both atopic and autoimmune disease may help uncover disease pathogenesis.

This large study benefited from its population-based design, precise incident RA classification, and adjustment for many confounders. However, it has several limitations. First, the epidemiology of allergic conditions may not generalise outside of its geographical area, as allergic diseases vary significantly by geographical area. ${ }^{38}$ Second, as a case-control study, it may be susceptible to recall bias, artificially increasing the observed associations. Third, the exposures in this study were self-reported and may therefore be prone to misclassification. We also had no information on childhood-onset versus adult-onset allergies. Fortunately, however, self-reported allergic diseases such as atopic dermatitis have high validity, ${ }^{39}$ and the prevalence of allergic diseases in this study is similar to published prevalences in the general population. ${ }^{3840} 41$ Fourth, sample size was too small to study each of the 59 allergic conditions separately, including some specific food allergies of interest such as nuts or eggs. Fifth, residual confounding (eg, asthma) is possible. Finally, multiple hypotheses were tested in this study, though all were based on a prespecified study protocol.

In conclusion, this study of incident RA found that most allergies overall are not associated with RA, but also that several atopic diseases including animal dander allergy, atopic dermatitis, and allergic rhinitis are associated with increased risk of RA.

Twitter Vanessa L Kronzer @KronzerMD, Cynthia S Crowson @CrowsonCindy and Marie Holmqvist @marie_holmqvist

Contributors All authors were involved in the drafting and critical review of the manuscript and approved the final version for submission. VK was responsible for overall content as the guarantor. HW, LA, LK, MH and JA were involved in acquisition of the data. VK, $\mathrm{HW}, \mathrm{MH}$ and JA were involved with the conception or design of the work. VK, HW, CC and MH were involved with the analysis of the data in the manuscript. All authors were involved with the interpretation of data in the manuscript. All authors agreed to be accountable for all aspects of the work and attest to the accuracy and integrity of the work.

Funding Funding for this project was provided by the RRF Resident Research Preceptorship (VK), the Mayo Clinic-Karolinska Institutet Collaborative Travel Grant Award (VK and MH), the Swedish Research Council (JA), the Swedish Heart Lung Foundation (JA), the Region-Stockholm ALF (JA) and The Nordic Research Council/ Vinnova (JA).

Competing interests None declared.

Patient consent for publication Not applicable.

Ethics approval This study involves human participants and was approved by Karolinska Insititutet Ethics Committee approval: (EPN 96-174, 2006/476-31/4, 2007/889-31/2, 2007/1443-32). Participants gave informed consent to participate in the study before taking part.

Provenance and peer review Not commissioned; externally peer reviewed.

Data availability statement All data relevant to the study are included in the article or uploaded as online supplemental information.

Open access This is an open access article distributed in accordance with the Creative Commons Attribution Non Commercial (CC BY-NC 4.0) license, which permits others to distribute, remix, adapt, build upon this work non-commercially, and license their derivative works on different terms, provided the original work is properly cited, appropriate credit is given, any changes made indicated, and the use is non-commercial. See: http://creativecommons.org/licenses/by-nc/4.0/.

\section{ORCID iDs}

Vanessa L Kronzer http://orcid.org/0000-0002-7489-3134

Helga Westerlind http://orcid.org/0000-0003-3380-5342

Cynthia S Crowson http://orcid.org/0000-0001-5847-7475

Lars Klareskog http://orcid.org/0000-0001-9601-6186

Marie Holmqvist http://orcid.org/0000-0001-8996-5260

Johan Askling http://orcid.org/0000-0003-0433-0616

\section{REFERENCES}

1 Klareskog L, Stolt P, Lundberg K, et al. A new model for an etiology of rheumatoid arthritis: smoking may trigger HLA-DR (shared epitope)-restricted immune reactions to autoantigens modified by citrullination. Arthritis Rheum 2006;54:38-46.

2 Makrygiannakis D, Hermansson M, Ulfgren A-K, et al. Smoking increases peptidylarginine deiminase 2 enzyme expression in human lungs and increases citrullination in BAL cells. Ann Rheum Dis 2008;67:1488-92. 
3 Kronzer VL, Crowson CS, Sparks JA, et al. Investigating asthma, allergic disease, passive smoke exposure, and risk of rheumatoid arthritis. Arthritis Rheumatol 2019;71:1217-24.

4 Sheen $\mathrm{YH}$, Rolfes MC, Wi C-I, et al. Association of asthma with rheumatoid arthritis: a population-based case-control study. $J$ Allergy Clin Immunol 2018;6:219-26.

5 Hou Y-C, Hu H-Y, Liu I-L, et al. The risk of autoimmune connective tissue diseases in patients with atopy: a nationwide populationbased cohort study. Allergy Asthma Proc 2017;38:383-9.

6 Lai N-S, Tsai T-Y, Koo M, et al. Association of rheumatoid arthritis with allergic diseases: a nationwide population-based cohort study. Allergy Asthma Proc 2015;36:99-103.

7 Hemminki K, Li X, Sundquist J, et al. Subsequent autoimmune or related disease in asthma patients: clustering of diseases or medical care? Ann Epidemiol 2010;20:217-22

8 de Roos AJ, Cooper GS, Alavanja MC, et al. Personal and family medical history correlates of rheumatoid arthritis. Ann Epidemiol 2008;18:433-9.

9 Ford JA, Liu X, Chu SH, et al. Asthma, chronic obstructive pulmonary disease, and subsequent risk for incident rheumatoid arthritis among women: a prospective cohort study. Arthritis Rheumatol 2020;72:704-13.

10 Kronzer VL, Westerlind H, Alfredsson L, et al. Respiratory diseases as risk factors for seropositive and seronegative rheumatoid arthritis and in relation to smoking. Arthritis Rheumatol 2021;73:61-8.

11 Zeller M. Rheumatoid arthritis; food allergy as a factor. Ann Allergy 1949;7:200-5.

12 Catterall WE. Allergy and arthritis. Ann Rheum Dis 1977;36:594.

$13 \mathrm{Li} \mathrm{J}$, Yan $\mathrm{H}$, Chen $\mathrm{H}$, et al. The pathogenesis of rheumatoid arthritis is associated with milk or egg allergy. N Am J Med Sci 2016;8:40-6.

14 O'Driscoll BR, Milburn HJ, Kemeny DM, et al. Atopy and rheumatoid arthritis. Clin Allergy 1985;15:547-53.

15 Peskett SA, Platts-Mills TA, Ansell BM, et al. Incidence of atopy in rheumatic disease. J Rheumatol 1981;8:321-4.

16 Hartung AD, Bohnert A, Hackstein $\mathrm{H}$, et al. Th2-mediated atopic disease protection in Th1-mediated rheumatoid arthritis. Clin Exp Rheumatol 2003;21:481-4.

17 Stolt P, Bengtsson C, Nordmark B, et al. Quantification of the influence of cigarette smoking on rheumatoid arthritis: results from a population based case-control study, using incident cases. Ann Rheum Dis 2003;62:835-41.

18 Bengtsson C, Berglund A, Serra M-L, et al. Non-Participation in EIRA: a population-based case-control study of rheumatoid arthritis. Scand J Rheumatol 2010;39:344-6.

19 Arnett FC, Edworthy SM, Bloch DA, et al. The American rheumatism association 1987 revised criteria for the classification of rheumatoid arthritis. Arthritis Rheum 1988;31:315-24.

20 Aletaha D, Neogi T, Silman AJ, et al. 2010 rheumatoid arthritis classification criteria: an American College of Rheumatology/ European League against rheumatism collaborative initiative. Arthritis Rheum 2010;62:2569-81.

21 Concato J, Feinstein AR, Holford TR. The risk of determining risk with multivariable models. Ann Intern Med 1993;118:201-10.

22 Lindberg $\mathrm{H}$, Colliander $\mathrm{C}$, Nise L, et al. Are farming and animal exposure risk factors for the development of granulomatosis with polyangiitis? environmental risk factors revisited: a case-control study. J Rheumatol 2021;48:894-7.

23 Svensson J, Holmqvist M, Lundberg IE, et al. Infections and respiratory tract disease as risk factors for idiopathic inflammatory myopathies: a population-based case-control study. Ann Rheum Dis 2017;76:1803-8.

24 Choi HK, Nguyen U-S, Niu J, et al. Selection bias in rheumatic disease research. Nat Rev Rheumatol 2014;10:403-12.

25 Jeong HE, Jung S-M, Cho S-I. Association between rheumatoid arthritis and respiratory allergic diseases in Korean adults: a propensity score matched case-control study. Int J Rheumatol 2018;2018:1-8.

26 Pedersen M, Jacobsen S, Klarlund M, et al. Environmental risk factors differ between rheumatoid arthritis with and without autoantibodies against cyclic citrullinated peptides. Arthritis Res Ther 2006;8:R133.

27 Hilliquin P, Allanore Y, Coste J, et al. Reduced incidence and prevalence of atopy in rheumatoid arthritis. Results of a case-control study. Rheumatology 2000;39:1020-6.

28 Reckner Olsson A, Skogh T, Wingren G. Comorbidity and lifestyle, reproductive factors, and environmental exposures associated with rheumatoid arthritis. Ann Rheum Dis 2001;60:934-9.

29 Rudwaleit M, Andermann B, Alten R, et al. Atopic disorders in ankylosing spondylitis and rheumatoid arthritis. Ann Rheum Dis 2002;61:968-74.

30 Olsson AR, Wingren G, Skogh T, et al. Allergic manifestations in patients with rheumatoid arthritis. APMIS 2003;111:940-4.

31 Karsh J, Chen Y, Lin M, et al. The association between allergy and rheumatoid arthritis in the Canadian population. Eur J Epidemiol 2005;20:783-7.

32 Schmitt J, Schwarz K, Baurecht H, et al. Atopic dermatitis is associated with an increased risk for rheumatoid arthritis and inflammatory bowel disease, and a decreased risk for type 1 diabetes. J Allergy Clin Immunol 2016;137:130-6.

33 Maas T, Nieuwhof C, Passos VL, et al. Transgenerational occurrence of allergic disease and autoimmunity: general practice-based epidemiological research. Prim Care Respir J 2014;23:14-21.

34 Lee S-H, Lee E-B, Shin E-S, et al. The interaction between allelic variants of CD86 and CD40LG: a common risk factor of allergic asthma and rheumatoid arthritis. Allergy Asthma Immunol Res 2014:6:137-41.

35 Frieri M. Neuroimmunology and inflammation: implications for therapy of allergic and autoimmune diseases. Ann Allergy Asthma Immunol 2003;90:34-40.

36 Murdaca G, Greco M, Tonacci A, et al. IL-33/IL-31 axis in immunemediated and allergic diseases. Int J Mol Sci 2019;20. doi:10.3390/ ijms20235856. [Epub ahead of print: 22 Nov 2019].

37 Bantz SK, Zhu Z, Zheng T. The atopic March: progression from atopic dermatitis to allergic rhinitis and asthma. J Clin Cell Immunol 2014;5.

38 Bylund S, Kobyletzki LB, Svalstedt M, et al. Prevalence and incidence of atopic dermatitis: a systematic review. Acta Derm Venereol 2020;100:adv00160.

39 Silverberg JI, Chiesa Fuxench ZC, Gelfand JM, et al. Content and construct validity, predictors, and distribution of self-reported atopic dermatitis severity in US adults. Ann Allergy Asthma Immunol 2018;121:729-34.

40 Meltzer EO, Blaiss MS, Derebery MJ, et al. Burden of allergic rhinitis: results from the pediatric allergies in America survey. J Allergy Clin Immunol 2009;124:S43-70.

41 Zuidmeer L, Goldhahn K, Rona RJ, et al. The prevalence of plant food allergies: a systematic review. J Allergy Clin Immunol 2008;121:1210-8. 